\title{
Inheritance of Capsule and the Manner of Cell-Wall Formation in Bacillus anthracis
}

\author{
By G. G. MEYNELL AND A. M. LAWN \\ Guinness-Lister Research Unit and the Department of Electron Microscopy, \\ Lister Institute of Preventive Medicine, Chelsea Bridge Road, London, S.W.1
}

(Received 5 February 1965 )

\section{SUMMARY}

During growth in broth, Bacillus anthracis strain $2160 \mathrm{~s}$ became capsulated only towards the end of exponential growth. An inoculum of fully capsulated organisms formed chains which were non-capsulated save at their tips and at occasional junctions between neighbouring cells. This appearance suggested that new non-capsulated wall was synthesized at the equator of each organism but not at the poles or throughout the existing wall.

\section{INTRODUCTION}

Although the synthesis of capsular material by bacteria has been studied extensively, there do not appear to be any reports of the fate of capsules once they have been formed. In many species capsule formation is determined by the growth phase of the culture (Meynell, 1961) and may therefore be suppressed although growth occurs. Thus Meynell \& Meynell (1965) found Bacillus anthracis was not capsulated until the end of the phase of exponential growth, and the present paper shows that when fully capsulated organisms divide after subculture to fresh broth, their capsules are partitioned amongst their progeny. This is believed to result from the way in which new cell wall is synthesized.

\section{METHODS}

Bacteria and culture media. The organism examined is a mutant (D31) of Bacillus anthracis 2160 s (Meynell \& Meynell, 1964) which differs from its parent by forming capsules in air. It was grown overnight on a shaker in $2 \mathrm{ml}$. medium containing (g./100 ml.): 'Lab-Lemco', 0.03; Tryptone (Oxoid), 0.07; peptone (Oxoid), 0.3; $\mathrm{NaCl}, 0.23$; dissolved in distilled water and adjusted to $\mathrm{pH} 7 \cdot 2$. The overnight culture of fully capsulated organisms was diluted 1/15-1/30 in the same medium or in $1 \%(\mathrm{w} / \mathrm{v})$ acid-hydrolysed casein $+1 \%(\mathrm{w} / \mathrm{v})$ glucose in $0.05 \mathrm{M}$-phosphate buffer (pH 7.2), supplemented with tryptophan and adenine (each $20 \mu \mathrm{g} . / \mathrm{ml}$.) and thiamine $\left(2 \mu \mathrm{g} . / \mathrm{ml}\right.$.). Cultures were grown in air at $37^{\circ}$ in a $250 \mathrm{ml}$. conical flask, with either $30 \mathrm{ml}$. medium with gentle shaking or $5 \mathrm{ml}$. medium without shaking.

Staining. Most of the films were examined after combined positive and negative staining by carbol fuchsin and nigrosin. Two lines about $1 \mathrm{~cm}$. apart were drawn with grease pencil across a slide and a drop of culture placed between them, mixed with a drop of buffered formaldehyde $(40 \%, \mathrm{w} / \mathrm{v}$, formaldehyde solution diluted $1 / 40$ in $0.05 \mathrm{M}$-glycerophosphate buffer $\mathrm{pH} \mathrm{7.2)}$, and allowed to dry in air. Positive staining was done with Ziehl-Neelsen carbol fuchsin (Mackie \& McCartney's 
Handbook, 1960) freshly diluted 1/10 in distilled water. After $1 \mathrm{~min}$., the film was washed with flowing tap water and gently blotted. One drop of $5 \%(w / v)$ nigrosin solution was added and spread over the film by drawing a wire across the drop. The slide was dried by standing it on edge on blotting paper. The nigrosin used was aqueous Nigrosin, batch no. 1062, received in 1962 from George T. Gurr Ltd., 136/140 New King's Road, London, S.W.6. A more recent batch (no. 1552) which was blacker and more viscous decolorized the carbol fuchsin.

Electron microscopy. Organisms were collected by centrifugation and washed in $0.4 \%(\mathrm{w} / \mathrm{v})$ formaldehyde solution in distilled water. A drop of bacterial suspension was mixed with a drop of dilute nigrosin (batch no. 2062 diluted about 1/4 with distilled water) on a copper grid bearing a Formvar + carbon supporting film. The surplus fluid was drained with filter paper and the grid dried in air. Specimens were examined in a Phillips EM 200 microscope.

\section{RESULTS}

Organisms were examined in wet indian ink films, in heat-fixed films stained by M'Fadyean's polychrome methylene blue, and in formaldehyde-fixed films stained by carbol fuchsin + nigrosin. Most of the observations were made by the last method; all three gave similar results. M'Fadyean's stain was never completely satisfactory. The relation between capsule and the individual organisms in a chain was not shown as clearly as in indian ink preparations or by the carbol fuchsin+ nigrosin stain, and the red component was found to be unstable in one sample of the stain as it eventually lost the power to stain the capsule at all. In films, the red colour always faded after a few hours under immersion oil. Indian ink films had to be examined immediately because particles of ink soon accumulated in masses on the organisms. The carbol fuchsin + nigrosin stain had none of these disadvantages and was convenient for studies of growth since the immediate fixation of samples by formaldehyde allowed more accurate timing.

The results of electron microscopy after negative staining with nigrosin were qualitatively similar to those observed in the light microscope with stained specimens.

A mixture of fully capsulated and non-capsulated organisms stained by carbol fuchsin + nigrosin is shown in Pl. 1, fig. 1. In the original film, the bodies of the organisms appeared a brilliant red, the capsules were white, and the background had the bluish black colour typical of nigrosin.

Bacillus anthracis $2160 \mathrm{~s}$ forms chains of 2 to 4 organisms in overnight culture and up to 50 organisms during exponential growth. With an inoculum of fully capsulated organisms like those shown in $\mathrm{Pl}$. 1, figs. 1, 5 the chains formed after incubation for about $3 \mathrm{hr}$ nearly always had blobs of capsule at their ends and at various intermediate points along their length which invariably overlapped the junctions between neighbouring organisms (Pl. 1, figs. 2-4). Occasionally a fully capsulated organism was seen at the end, or less often in the middle, of a chain. In all cases, capsule extended over the union between the fully capsulated organism and its non-capsulated neighbour. Less commonly, a chain had a blob of capsule at one end only (Pl. 1, fig. 6). This was rare 1.5-2 hr after inoculation, but became more common with longer incubation. 


\section{DISCUSSION}

The most straightforward interpretation of our results is that when capsulated organisms of this strain were subcultured to fresh broth and multiplied in conditions which prevented further capsule formation, their capsules were partitioned amongst their progeny in a manner determined by the way in which new cell-wall was made. A less plausible interpretation is that the capsule was not attached to the bacterium but formed a shell around it which split in half at division, irrespective of how new cell-wall was formed. Various patterns of cell-wall synthesis have been suggested for bacteria. Of the four shown schematically in Fig. 1, one pattern presupposes a
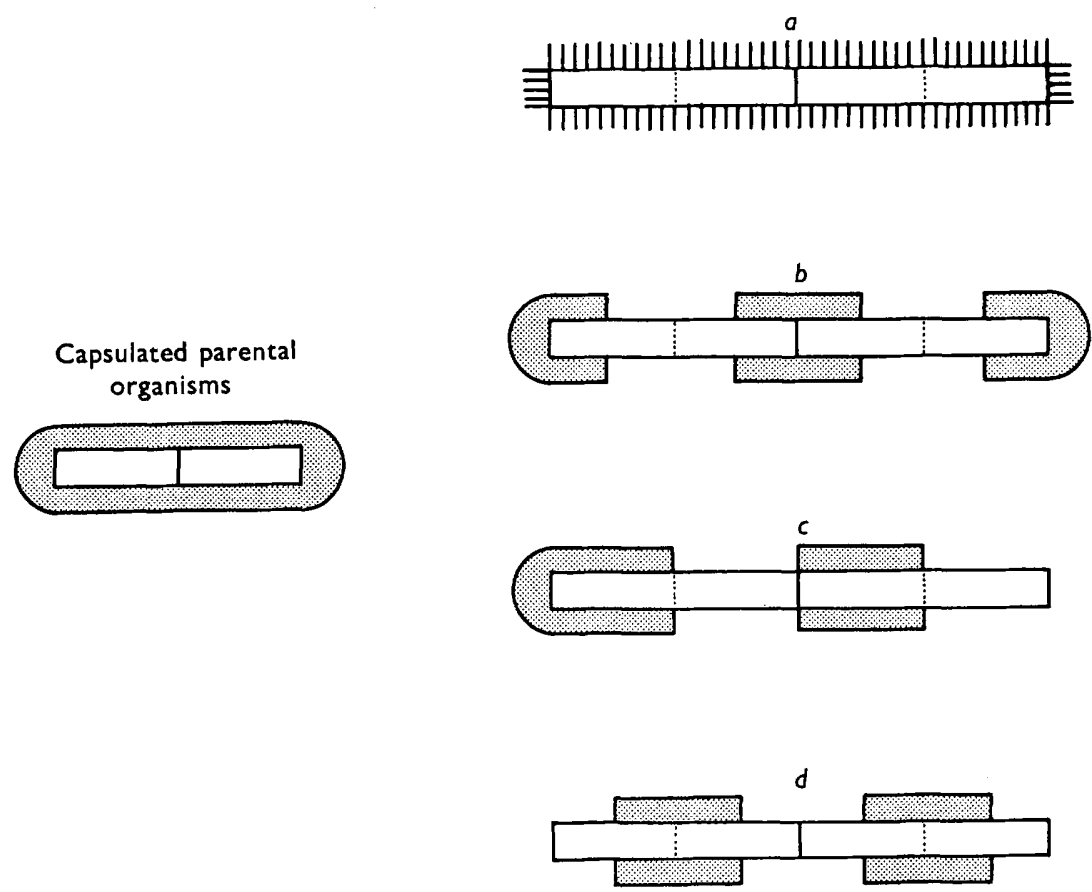

Fig. 1. Cell-wall synthesis and its effect on the inheritance of capsule. A pair of fully capsulated organisms is shown on the left and four possible types of daughters on the right. New transverse septa are shown by dotted lines. Assuming wall and capsule to be associated, diffuse synthesis of new wall leads to $(a)$; equatorial growth to $(b)$; unipolar growth to $(c)$-two other permutations might occur but all produce daughters that are either non-capsulated or fully capsulated; and bipolar growth leads to $(d)$. Only equatorial growth accounts for the appearances shown in Pl. 1.

diffuse process in which new wall appears uniformly amongst pre-existent wall ('intercalation' : Quadling \& Stocker, 1962; May, 1963), and the other three patterns some form of localized process in which new wall is synthesized at one point in the parent, as in budding, so that new and old wall are sharply separated in the progeny. The anthrax capsule is clearly inherited not diffusely but in blocks, so that cell-wall synthesis by intercalation is excluded if the parental wall and its capsule remain 
joined. As regards localized processes, the main possibilities are equatorial growth as in Streptococcus faecalis (Chung, Hawirko \& Isaac, 1964b) and polar growth (Bisset, 1956; Bisset \& Pease, 1957). Direct observation of individual organisms shows, however, that new cell-wall is not always formed in precisely the same way at each generation (e.g. fig. $5 c$ in Chung et al. 1964b).

Equatorial growth accounts for the appearance of the chains shown in Pl. 1, figs. 2-4. The inoculum usually consist of pairs of fully capsulated organisms (PI. 1, fig. 1) whose progeny on division remain linked together. The original cellwall of the parental organisms and its associated capsule are presumably partitioned between daughters at the first division, as shown in Fig. 1 and continue to be inherited together at later divisions. This will result in the invariable presence of capsule at the extremities of the terminal organisms of the chain and, when the chain originated from $n$ organisms, at $n-1$ points along the chain. At these points the capsule must lie across the junction of neighbouring organisms but never round their equators. This is precisely what was observed. When the capsule was present at one point along the chain, there were often unequal numbers of organisms between it and the ends, presumably because of asynchrony of division. It is to be expected from equatorial growth that half of each terminal organism will be capsulated, on the average, but in fact only about one-fifth had capsule. The reason for this discrepancy is partly due to the fact that the multiplying bacteria were longer than the organisms in the stationary phase, but the residues of capsules may collapse upon themselves or the staining methods may underestimate their size.

Polar growth is incompatible with the observed results, whatever form it takes (see Fig. 1). If unipolar, the process is equivalent to budding and results in daughter organisms with either the full complement of capsule or none at all; if bipolar, capsule is confined to junctions within the chain and is absent from the ends. The occasional chains in young cultures which lacked capsule at one end (Pl. 1, fig. 6) might have arisen in this way, but might equally well have been formed by breakage of chains like those shown in Pl. 1, figs. 2-4, an explanation supported by their increasing frequency as growth continued.

Cell-wall formation can be studied more directly by fluorescence microscopy, using labelled antibody in the method introduced by Cole \& Hahn (1962). The growth of Bacillus anthracis has not been examined in this way but that of two related species, B. cereus and B. megaterium, was investigated by Chung, Hawirko \& Isaac $(1964 a)$. In neither of these latter species did intercalation occur; both grew equatorially and $B$. megaterium also grew at the poles (see fig. $2 b$ in Chung et al. 1964a). Photographs of the clones which were increasing by equatorial growth resemble those shown here in Pl. 1, figs. 2-4, with patches of fluorescence which indicated the position of parental cell-wall at the ends of chains and at intermediate points, similar to the distribution of inherited pieces of capsule.

\section{REFERENCES}

Bisset, K. A. (1956). Cellular organization in bacteria. Symp. Soc. gen. Microbiol. 6, 1. Bisset, K. A. \& Pease, P. (1957). The distribution of flagella in dividing bacteria. J.gen. Microbiol. 16, 382.

Chung, K. L., Hawirko, R. Z. \& IsaAc, P. K. (1964a). Cell wall replication. I. Cell wall growth of Bacillus cereus and Bacillus megaterium. Can. J. Microbiol. 10, 43. 
Journal of General Microbiology, Vol. 39, No. 3

Plate 1
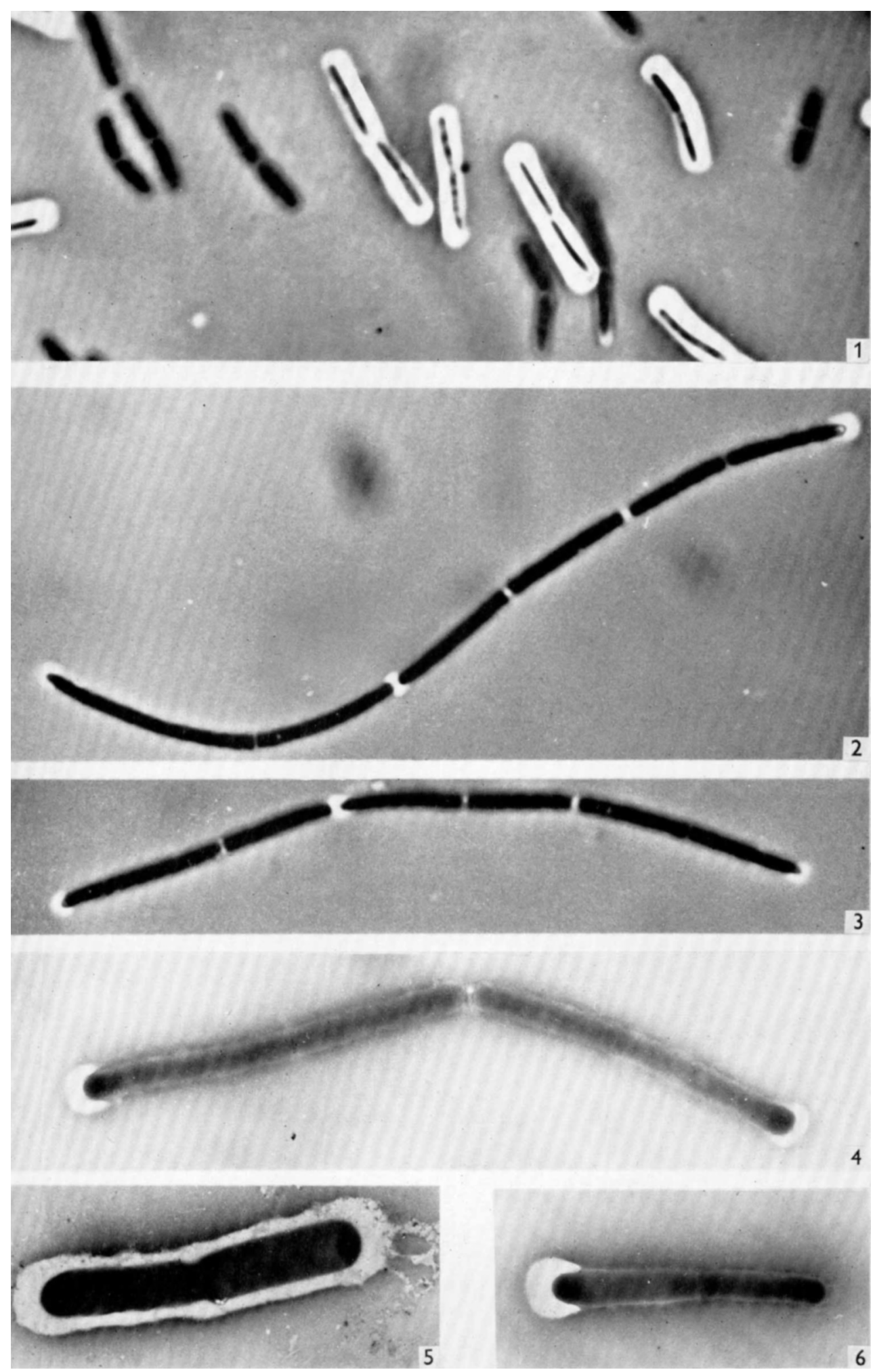

G. G. MEYNELL AND A. M. LAWN

(Facing p. 426) 
Chung, K. L., Hawirko, R. Z. \& IsaAc, P. K. (1964b). Cell wall replication. II. Cell wall growth and cross wall formation of Escherichia coli and Streptococcus faecalis. Can. J. Microbiol. 10, 473.

Cole, R. M. \& HahN, J. J. (1962). Cell wall replication in Streptococcus pyogenes. Science, $135,722$.

Mackie and McCartney's Handbook of Bacteriology (1960). 10th ed. Ed. by R. Cruickshank Edinburgh and London: E. and S. Livingstone Ltd.

MAY, J. W. (1963). The distribution of cell wall label during growth and division of Salmonella typhimurium. Exp. Cell Res. 31, 217.

Meynell, E. \& Meyneli, G. G. (1964). The roles of serum and carbon dioxide in capsule formation by Bacillus anthracis. J. gen. Microbiol. 34, 153.

Meynell, G. G. (1961). Phenotypic variation and bacterial infection. Symp. Soc. gen. Microbiol. 11, 174.

Meyneld, G. G. \& Meynel.L, E. W. (1965). Biosynthesis of the polypeptide capsule and the cell wall of Bacillus anthracis. J. gen. Microbiol. 39, vii.

Quadung, C. \& Stocker, B. A. D. (1962). An environmentally-induced transition from the flagellated to the non-flagellated state in Salmonella typhimurium: the fate of parental flagella at cell division. J. gen. Microbiol. 20, 257.

\section{EXPLANATION OF PLATE 1}

Bacillus anthracis: a mutant (D 31) of B. anthracis 2160 s (Meynell \& Meynell, 1964). The organisms in figs. $2,3,4$, and 6 came from growing cultures inoculated 1.5 to $3 \mathrm{hr}$ earlier with fully capsulated organisms.

Fig. 1. Fully capsulated organisms from an overnight culture mixed with non-capsulated organisms. Carbol fuchsin + nigrosin.

Figs. 2, 3. Chains with capsules at each end and at one or more points along their length. Carbol fuchsin + nigrosin.

Fig. 4. Chain with capsules at both ends. Nigrosin negative staining. Electron micrograph.

Fig. 5. Capsulated organisms from an overnight culture. Nigrosin negative staining. Electron micrograph.

Fig. 6. A pair of organisms with capsule at one end only. Nigrosin negative staining. Electron micrograph. 